Nota científica

\title{
Primer registro de la sanguijuela introducida Barbronia weberi (Annelida: Clitellata) en Nuevo León, México
}

\author{
First record of the introduced leech Barbronia weberi (Annelida: Clitellata) in Nuevo Leon, Mexico \\ Uriel Garduño-Montes de Oca ${ }^{\mathrm{a}}$, Gisela Martínez-Flores ${ }^{\mathrm{b}}$, Samantha Contreras-Mirón ${ }^{\mathrm{b}}$, \\ Jossué Jiménez-Armenta ${ }^{\mathrm{b}}$ y Alejandro Oceguera-Figueroa ${ }^{\mathrm{b}, *}$

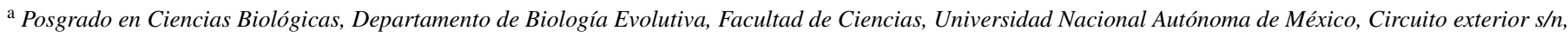 \\ Ciudad Universitaria-Copilco, Coyoacán, 04510, Coyoacán, Ciudad de México, México \\ ${ }^{\mathrm{b}}$ Laboratorio de Helmintología, Instituto de Biología, Universidad Nacional Autónoma de México, Apartado postal 70153, Ciudad Universitaria-Copilco, \\ Coyoacán, 04510, Ciudad de México, México \\ Recibido el 18 de noviembre de 2015; aceptado el 7 de marzo de 2016 \\ Disponible en Internet el 22 de agosto de 2016
}

\begin{abstract}
Resumen
Se documenta la presencia de la sanguijuela introducida Barbronia weberi en el río San Juan, Monterrey, Nuevo León, México, que corresponde al segundo registro de esta especie de agua dulce en el país. La identificación de los ejemplares se realizó con base en caracteres morfológicos y moleculares del gen citocromo $c$ oxidasa, subunidad I.

Derechos Reservados () 2016 Universidad Nacional Autónoma de México, Instituto de Biología. Este es un artículo de acceso abierto distribuido bajo los términos de la Licencia Creative Commons CC BY-NC-ND 4.0.
\end{abstract}

Palabras clave: Hirudinea; Citocromo $c$ oxidasa subunidad I; Determinación molecular

\section{Abstract}

The presence of the introduced leech Barbronia weberi in the San Juan river, Monterrey, Nuevo León, Mexico, is reported. This is the second record of the freshwater species in the country. The identification of the specimens was carried out considering morphological and molecular characters of the gene cytochrome oxidase subunit I.

All Rights Reserved (C 2016 Universidad Nacional Autónoma de México, Instituto de Biología. This is an open access item distributed under the Creative Commons CC License BY-NC-ND 4.0.

Keywords: Hirudinea; Cytochrome oxidase subunit I; Molecular identification

Existe un amplio registro de los efectos ecológicos y económicos causados por la introducción de especies exóticas, por lo que la identificación taxonómica es crucial para la toma de decisiones encaminadas a mitigar estos efectos (Jeschke et al., 2014; Molnar, Gamboa, Revenga y Spalding, 2008). Barbronia weberi Blanchard, 1897, es una sanguijuela dulceacuícola perteneciente a la familia Salifidae Johansson, 1910, cuyos

\footnotetext{
* Autor para correspondencia.

Correo electrónico: aoceguera@ib.unam.mx (A. Oceguera-Figueroa).

La revisión por pares es responsabilidad de la Universidad Nacional Autónoma de México.
}

miembros son depredadores de invertebrados acuáticos, y presentan una distribución nativa que abarca varios países de Asia (Genoni y Fazzone, 2008). Su introducción accidental ha sido registrada en Nueva Zelanda, Australia, Inglaterra, Alemania, Italia, Brasil, Estados Unidos, Costa Rica, México y Sudáfrica (Genoni y Fazzone, 2008; Oceguera-Figueroa, León-Règagnon y Siddall, 2005). El 14 de julio de 2015 se recolectaron 2 ejemplares de hirudíneos en el río San Juan, frente a la entrada de la Cueva de los Murciélagos, municipio de Santiago, Nuevo León, México (2525’59.96” N, 10006’49.79” O; 425 m snm). Las sanguijuelas fueron procesadas conforme a lo descrito por Oceguera-Figueroa et al. (2005) y depositadas en la Colección 

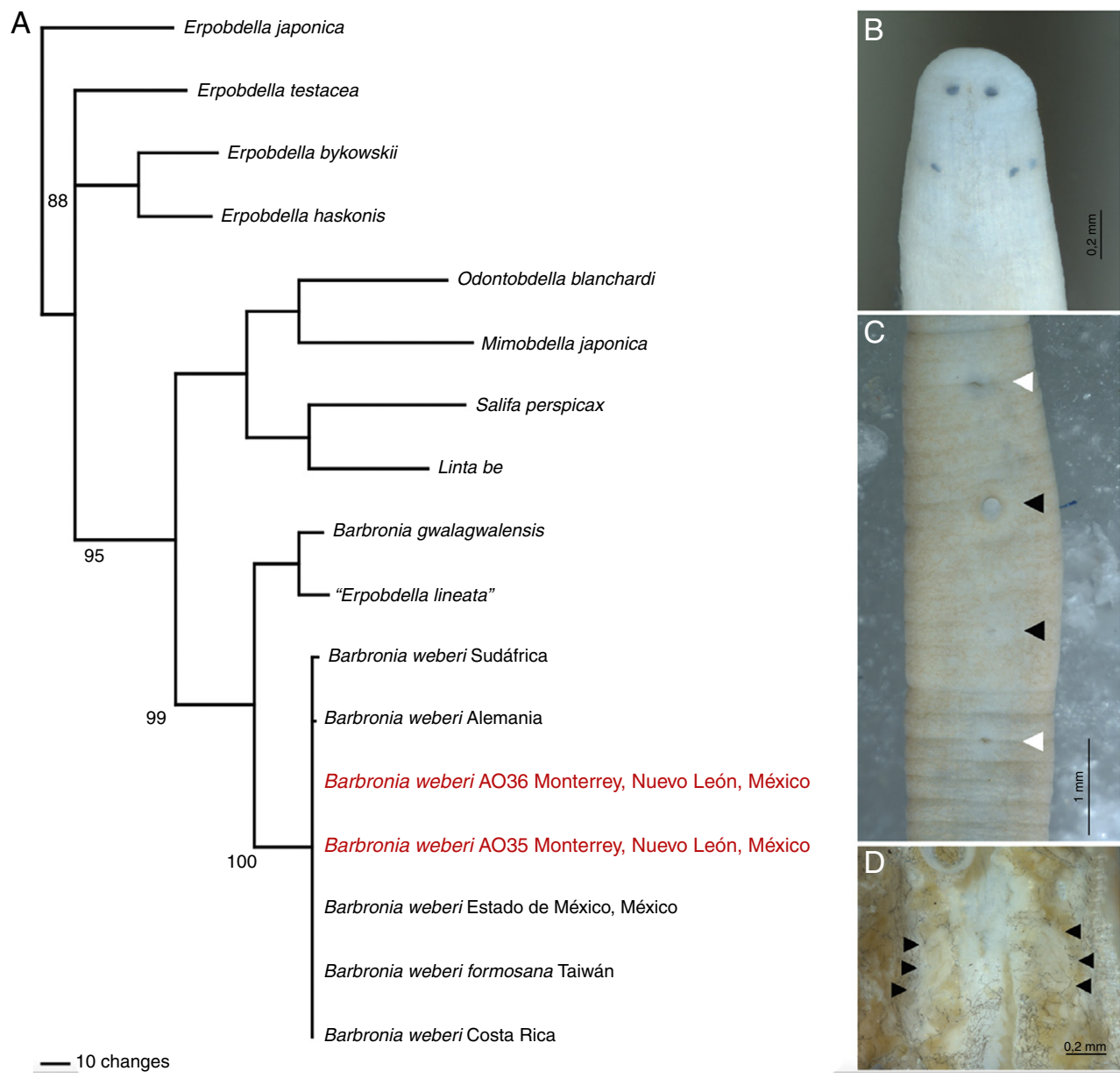

Figura 1. A) Árbol de consenso estricto. Códigos de acceso de GenBank ${ }^{\circledR}$ después de los nombres. En rojo se señalan las muestras recolectadas en este trabajo; B) Barbronia weberi, manchas oculares, un par anterior y 2 pares posteriores; C) región ventral del clitelo: triángulos blancos señalan gastroporos, triángulos negros señalan el gonoporo masculino y el femenino; D) ciegos laterales en la región posterior del buche señalados con flechas.

Nacional de Helmintos con el número de catálogo 9908. Se obtuvieron secuencias del gen mitocondrial citocromo $c$ oxidasa (COXI) siguiendo el protocolo descrito en Oceguera-Figueroa et al. (2005). Las secuencias de ADN se alinearon en el programa Muscle (Edgar, 2004) junto con otros representantes de hirudíneos (tabla 1). Se realizó un análisis de parsimonia y se calcularon distancias genéticas (K2P) en el programa PAUP* 4.0b10 (Swofford, 2000) siguiendo los métodos descritos en Oceguera-Figueroa et al. (2005). El análisis resultó en 20 árboles óptimos de 606 pasos. El árbol de consenso estricto se muestra en la figura 1A. Los ejemplares tienen un largo máximo de $19 \mathrm{~mm}$ y un ancho máximo de $1 \mathrm{~mm}$. Presentan un color rojo pálido, ligeramente más claro en la superficie ventral y un par de manchas oculares en posición labial y 2 pares bucales (fig. 1B). Presentan un par de poros en la línea media ventral, uno anterior al gonoporo masculino y uno posterior al gonoporo femenino (fig. 1C). La región de la boca con 3 pares de estiletes, la faringe es larga y muscular. El buche es tubular, y en la región posterior presenta un par de ciegos laterales con distribución posterior (fig. 1D). Estos caracteres morfológicos son consistentes con los descritos para B. weberi por Lai y Chen (2010). Todas las muestras asignadas morfológicamente a $B$. weberi forman un grupo fuertemente apoyado (Bootstrap $=100 \%)$ con variación molecular prácticamente nula $(<0.8 \%)$. Tanto la evidencia morfológica como molecular señalan que los ejemplares recolectados en el estado de Nuevo León corresponden a $B$. weberi. Con el presente registro son 2 localidades en México (Nuevo León y Morelos) en las que se ha registrado a esta especie de sanguijuela, las cuales se encuentran separadas entre sí por más de $750 \mathrm{~km}$ y pertenecen a provincias biogeográficas distintas (Morrone, 2005). Se desconoce el impacto que esta especie introducida pueda tener sobre las poblaciones de invertebrados dulceacuícolas de los que se alimenta y, de igual forma, se desconoce si por competencia puedan estar desplazando a las sanguijuelas macrófagas nativas del género Erpobdella. Se sugiere que esta especie pudo hacer sido introducida accidentalmente por el comercio de peces de ornato, una práctica común en el área. La presencia de B. weberi en los cuerpos de agua dulce de Nuevo León se suma a una amplia lista de especies invasoras como el acocil rojo Procambarus clarkii (Hernández et al., 2008), el bagre Ictalurus punctatus (DOF, 2012), la trucha arcoíris Oncorhynchus mykiss (Álvarez-del Villar, 1970), la rana toro Lithobates catesbeianus (Kellogg, 1932) y el caracol Melanoides tuberculata (Facon et al., 2003).

Este trabajo se realizó gracias a los fondos del programa de apoyo para el PAPIIT-UNAM IA204114 de AO-F y de la Red 
Tabla 1

Taxones utilizados con fines comparativos para la identificación molecular de Barbronia weberi de Nuevo León, México, con localidades de recolecta y claves de acceso de GenBank ${ }^{\circledR}$.

\begin{tabular}{|c|c|c|c|}
\hline Taxón & Localidad & $\begin{array}{l}\text { Número de } \\
\text { GenBank }^{\circledR}\end{array}$ & $\begin{array}{l}\text { Referencia } \\
\text { bibliográfica }\end{array}$ \\
\hline $\begin{array}{r}\text { Erpobdella } \\
\text { japonica }\end{array}$ & Corea del Sur & AF116026 & $\begin{array}{l}\text { Apakupakul, } \\
\text { Siddall y } \\
\text { Burreson, } 1999\end{array}$ \\
\hline $\begin{array}{l}\text { Erpobdella } \\
\text { testacea }\end{array}$ & Francia & AF116027 & $\begin{array}{l}\text { Apakupakul } \\
\text { et al., } 1999\end{array}$ \\
\hline $\begin{array}{l}\text { Erpobdella } \\
\quad \text { bykowskii }\end{array}$ & Austria & DQ009667 & $\begin{array}{l}\text { Pfeiffer, Brenig } \\
\text { yKutschera, } \\
2005\end{array}$ \\
\hline $\begin{array}{l}\text { Erpobdella } \\
\text { haskonis }\end{array}$ & Alemania & DQ009668 & $\begin{array}{l}\text { Pfeiffer et al., } \\
2005\end{array}$ \\
\hline $\begin{array}{r}\text { Odontobdella } \\
\text { blanchardi }\end{array}$ & Japón & 697993305 & Nakano, 2014 \\
\hline $\begin{array}{l}\text { Mimobdella } \\
\text { japonica }\end{array}$ & Japón & AB761395 & Nakano, 2013 \\
\hline Salifa perspicax & Ruanda & HQ336342 & $\begin{array}{l}\text { Oceguera- } \\
\text { Figueroa et al., } \\
2010\end{array}$ \\
\hline Linta be & Madagascar & AY786460 & $\begin{array}{l}\text { Siddall et al., } \\
2007\end{array}$ \\
\hline $\begin{array}{l}\text { Barbronia } \\
\quad \text { gwalagwalensis }\end{array}$ & Sudáfrica & AY786455 & $\begin{array}{l}\text { Siddall et al., } \\
2007\end{array}$ \\
\hline Erpobdella lineata & Corea del Sur & KF966549 & $\begin{array}{l}\text { Artículo no } \\
\text { publicado }\end{array}$ \\
\hline Barbronia weberi & Sudáfrica & AY786457 & $\begin{array}{l}\text { Siddall et al., } \\
2007\end{array}$ \\
\hline Barbronia weberi & Alemania & DQ009666 & $\begin{array}{l}\text { Pfeiffer et al., } \\
2005\end{array}$ \\
\hline Barbronia weberi & $\begin{array}{l}\text { Nuevo León, } \\
\text { México }\end{array}$ & KU553102 & Presente trabajo \\
\hline Barbronia weberi & $\begin{array}{l}\text { Nuevo León, } \\
\text { México }\end{array}$ & KU553103 & Presente trabajo \\
\hline Barbronia weberi & $\begin{array}{l}\text { Morelos, } \\
\text { México }\end{array}$ & DQ235598 & $\begin{array}{l}\text { Oceguera- } \\
\text { Figueroa et al., } \\
2005\end{array}$ \\
\hline $\begin{array}{l}\text { Barbronia weberi } \\
\text { formosana }\end{array}$ & Hawái & AY786456 & $\begin{array}{l}\text { Borda y Siddall, } \\
2004\end{array}$ \\
\hline Barbronia weberi & Costa Rica & HQ336339 & $\begin{array}{l}\text { Oceguera } \\
\text { Figueroa, } \\
\text { Phillips, } \\
\text { Pacheco Chaves, } \\
\text { Reeves y } \\
\text { Siddall, } 2011\end{array}$ \\
\hline
\end{tabular}

CONACyT-MEXBOL. A. Jiménez-Marín y S. Guzmán Gómez (IB-UNAM) colaboraron en la obtención de secuencias de ADN y fotografías, respectivamente. Luis García de la CNHE-IB facilitó el uso de la colección.

\section{Referencias}

Apakupakul, K., Siddall, M. E. y Burreson, E. M. (1999). Higher level relationships of leeches (Annelida: Clitellata: Euhirudinea) based on morphology and gene sequences. Molecular Phylogenetics and Evolution, 12, 350-359.
Álvarez-del Villar, J. (1970). Peces mexicanos (claves). Serie de Investigación Pesquera. Estudio Núm. 1. Distrito Federal, México: Instituto Nacional de Investigaciones Biológico Pesqueras. Secretaría de Industria y Comercio.

Borda, E. y Siddall, M. E. (2004). Review of the evolution of life history strategies and phylogeny of the Hirudinida (Annelida: Oligochaeta). Lauterbornia, 52, 25.

Diario Oficial de la Federación (2012). Secretaría de Agricultura, Ganadería, Desarrollo Rural, Pesca y Alimentación. Acuerdo mediante el cual se aprueba la actualización de la Carta Nacional Acuícola. 6 de junio de 2012.

Edgar, R. C. (2004). MUSCLE: multiple sequence alignment with high accuracy and high throughput. Nucleic Acids Research, 32, 1792-1797.

Facon, B., Pointier, J., Glaubrecht, M., Poux, C., Jarne, P. y David, P. (2003). A molecular phylogeography approach to biological invasions of the New World by parthenogenetic thiarid snails. Molecular Ecology, 12, 3027-3039.

Genoni, P. y Fazzone, A. (2008). Barbronia weberi (R. Blanchard, 1897) (Hirudinea: Salifidae), an Asian leech species new to Italy. Aquatic Invasions, 3, 77-79.

Hernández, L., Maeda-Martínez, A. M., Ruiz-Campos, G., Rodríguez-Almaraz, G., Alonzo-Rojo, F. y Sainz, J. C. (2008). Geographic expansion of the invasive red crayfish Procambarus clarkii (Girard, 1852) (Crustacea: Decapoda) in Mexico. Biological Invasions, 10, 977-984.

Jeschke, J. M., Bacher, S., Blackburn, T. M., Dick, J. T., Essl, F., Evans, T., et al. (2014). Defining the impact of non-native species. Conservation Biology, $28,1188-1194$.

Kellogg, R. (1932). Mexican tailles amphibians in the United States National Museum. US National Museum Bulletin, 160, 1-224.

Lai, Y. T. y Chen, J. H. (2010). Leech fauna of Taiwan. Taipei: National Taiwan University Press.

Molnar, J. L., Gamboa, R. L., Revenga, C. y Spalding, M. D. (2008). Assessing the global threat of invasive species to marine biodiversity. Frontiers in Ecology and the Environment, 6, 485-492.

Morrone, J. J. (2005). Hacia una síntesis biogeográfica de México. Revista Mexicana de Biodiversidad, 76, 207-252.

Nakano, T. (2013). First record of Mimobdella japonica (Hirudinida: Arhynchobdellida: Salifidae) from Okinawajima Island, Ryukyu Islands, Japan, with a description of the specimens from the Ryukyu Islands. Species Diversity, 18, 99-103.

Nakano, T. (2014). A new quadrannulate species of Orobdella (Hirudinida, Arhynchobdellida, Orobdellidae) from central Honshu, Japan. Zookeys, 445, 57.

Oceguera-Figueroa, A., León-Règagnon, V. y Siddall, M. E. (2005). Phylogeny and revision of Erpobdelliformes (Annelida, Arhynchobdellida) from Mexico based on nuclear and mitochondrial gene sequences. Revista Mexicana de Biodiversidad, 72, 191-198.

Oceguera Figueroa, A., Phillips, A. J., Pacheco Chaves, B., Reeves, W. K. y Siddall, M. E. (2011). Phylogeny of macrophagous leeches (Hirudinea, Clitellata) based on molecular data and evaluation of the barcoding locus. Zoologica Scripta, 40, 194-203.

Pfeiffer, I., Brenig, B. y Kutschera, U. (2005). Molecular phylogeny of selected predaceous leeches with reference to the evolution of body size and terrestrialism. Theory in Biosciences, 124, 55-64.

Siddall, M. E., Trontelj, P., Utevsky, S. Y., Nkamany, M. y Macdonald, K. S. (2007). Diverse molecular data demonstrate that commercially available medicinal leeches are not Hirudo medicinalis. Proceedings of the Royal Society of London B: Biological Sciences, 274, 1481-1487.

Swofford, D. L. (2000). PAUP*. Phylogenetic Analysis Using Parsimony (*and other methods), version 4. Sunderland, Massachusetts: Sinauer Associates. 